\title{
Antigos argumentos, novos desafios: políticas públicas e o movimento antivacina
}

\author{
Old arguments, new challenges: public policies and the anti-vax movement \\ Viejos argumentos, nuevos retos: las políticas públicas y el movimiento antivacuna
}

Recebido: 28/10/2021 | Revisado: 06/11/2021 | Aceito: 06/11/2021 | Publicado: 11/11/2021

Leonardo Linhares Miler da Silva
ORCID: https://orcid.org/0000-0002-8301-682X
Universidade Católica de Petrópolis, Brasil
E-mail: leonardolinhares_miler@ hotmail.com
Raphaela Abud Neves
ORCID: https://orcid.org/0000-0002-8244-2923
Universidade do Estado do Rio de Janeiro, Brasil
E-mail: raphaelaabud@ @mail.com
Rodrigo Grazinoli Garrido
ORCID: https://orcid.org/0000-0002-6666-4008
Universidade Católica de Petrópolis, Brasil
E-mail: grazinoli.garrido@ gmail.com
Daniel Machado Gomes
ORCID: https://orcid.org/0000-0003-0615-1814
Universidade Católica de Petrópolis, Brasil
E-mail: daniel.machado@ ucp.br

\begin{abstract}
Resumo
Não constitui novidade a existência de desafios impostos pelo negacionismo científico às políticas públicas de imunização. Na história do Brasil, observa-se que o movimento antivacina esteve presente desde o século XIX, baseando-se em justificativas falaciosas que descredenciavam a ciência pelos mais diferentes argumentos jurídicos, morais e religiosos. O presente texto tem como objetivo interpretar a refutação às políticas públicas de combate à pandemia de coronavírus à luz dos eventos que resultaram na Revolta da Vacina. Para tanto, realizou-se uma revisão bibliográfica narrativa, delineada pela busca de artigos científicos e bibliografias nos principais repositórios eletrônicos internacionais. Os descritores utilizados para busca foram: vacinas, COVID-19, imunização, histórico, medidas sanitárias, negacionismo, movimentos antivacina e Brasil, além das suas respectivas traduções para a língua inglesa. Foi percebida uma linha de continuidade entre os discursos do passado e do presente que se opõem às medidas restritivas e à vacinação, seja pela alegação de defesa da liberdade individual, seja pela invocação de ideias obscurantistas. Conclui-se que a recusa arbitrária ao cumprimento de políticas públicas sanitárias configura exercício abusivo do direito de liberdade individual, por expor a sociedade a riscos graves e evitáveis.
\end{abstract}

Palavras-chave: Revolta da Vacina; COVID-19; Imunização; Medidas restritivas.

\begin{abstract}
The existence of challenges to public immunization policies imposed by scientific denialism is not new. In the history of Brazil, it is observed that the anti-vax movement has existed since the 19th century, based on fallacious justifications that have discredited science by the most different legal, moral, and religious arguments. This article aims to interpret the refutation of public policies to combat the coronavirus pandemic under the events that resulted in the Vaccine Revolt. To this end, a narrative literature review was conducted, outlined by searching for scientific articles and bibliographies in the main international electronic repositories. The descriptors used for the search were: vaccines, COVID-19, immunization, history, sanitary measures, denialism, anti-vax movements, and Brazil. A line of continuity was perceived between past and present discourses opposing restrictive measures and vaccination, either by claiming to defend individual freedom or by invoking obscurantist ideas. We have concluded that the arbitrary refusal to comply with public health policies constitutes an abusive exercise against the right to individual freedom, by exposing society to serious and avoidable risks.
\end{abstract}

Keywords: Vaccine Revolt; COVID-19; Immunization; Restrictive measures.

\section{Resumen}

La existencia de desafíos impuestos por el negacionismo científico a las políticas públicas de inmunización no es nueva. En la historia de Brasil, se observa que el movimiento antivacuna ha estado presente desde el siglo XIX, basándose en justificaciones falaces que desacreditaban a la ciencia a través de los más diversos argumentos jurídicos, morales y religiosos. El presente texto tiene como objetivo interpretar la refutación de las políticas públicas de lucha contra el coronavirus a la luz de los acontecimientos que dieron lugar a la Revolta da Vacina. Para ello, se llevó a cabo una revisión bibliográfica narrativa, esbozada mediante la búsqueda de artículos científicos y bibliográficos en 
los principales repositorios electrónicos internacionales. Los descriptores utilizados para la búsqueda fueron: vacunas, COVID-19, inmunización, historia, medidas sanitarias, negacionismo, movimientos antivacuna y Brasil, así como sus respectivas traducciones al inglês. Se percibe una línea de continuidad entre los discursos pasados y presentes que se oponen a las medidas restrictivas y a la vacunación, ya sea alegando la defensa de la libertad individual o invocando ideas oscurantistas. Se concluye que la negativa arbitraria a cumplir con las políticas de salud pública configura un ejercicio abusivo del derecho a la libertad individual, al exponer a la sociedad a riesgos graves y evitables.

Palabras clave: Revolta da Vacina; COVID-19; Inmunización; Medidas restrictivas.

\section{Introdução}

A procura por saúde e melhor qualidade de vida é um aspecto marcante nas civilizações. Por outro lado, durante milênios, indivíduos perderam a vida sem o conhecimento das possíveis causas ou, em muitos casos, quando a razão era estabelecida, não havia métodos de controle. Assim, devido à necessidade de reduzir a morbimortalidade por doenças, em especial as transmissíveis, responsáveis por endemias locais e grandes epidemias, o avanço do conhecimento médico levou ao desenvolvimento das vacinas.

A vacina é um composto formado pela suspensão de micro-organismos patogênicos mortos, atenuados ou de fragmentos do agente, capaz de conduzir à imunização do indivíduo (Jesus et al., 2021). Por esse motivo, elevam-se significativamente as chances de uma determinada doença não ser contraída de forma ativa, tornando a vacina uma das maiores conquistas científicas da humanidade.

Apesar da comprovada eficiência quando se trata de reduzir o número de doentes e mortos, bem como erradicar doenças infecto-contagiosas, não é raro que períodos sombrios, atrelados aos movimentos contra o sanitarismo, coloquem em dúvida a eficácia do processo de vacinação no Brasil ou em qualquer parte do mundo. Tal fato pode ser atestado pelos movimentos negacionistas e antivacinação, observados em meio à pandemia de COVID-19, que vêm colocando à prova os esforços realizados por inúmeras instituições, cientistas e outros profissionais da saúde. Dentro desse cenário, o presente texto tem o objetivo de interpretar a refutação às políticas públicas de combate à pandemia de coronavírus à luz dos eventos que resultaram na Revolta da Vacina.

\section{Metodologia}

O trabalho foi realizado por meio de uma revisão bibliográfica narrativa, delineada pela busca de artigos científicos e bibliografias com enfoque no histórico das vacinas nas plataformas eletrônicas National Center for Biotechnology Information (NCBI), Scientific Eletronic Library (Scielo) e Google Acadêmico. Os descritores utilizados para busca foram: vacinas, COVID-19, imunização, histórico, medidas sanitárias, negacionismo, movimentos antivacina e Brasil, além das suas respectivas traduções para a língua inglesa. A metodologia utilizada possui caráter qualitativo, com o intuito de obter um apanhado de informações robusto e atual de dados preferencialmente descritivos, onde a análise destes, interpretação e correlação de informações seguem uma linha crítica e indutiva (Pereira et al., 2018). Os trabalhos selecionados variam do período de 1990 a 2021, dos quais os materiais mais antigos foram utilizados para fundamentação e contextualização histórica. Sendo assim, os estudos que não apresentavam enfoque na temática escolhida foram desconsiderados.

\section{Resultados e Discussão}

Por meio de estudos questionáveis, artigos duvidosos e, principalmente, através de fake news nas redes sociais, os antivacinas difundem o conceito de que a vacinação não é necessária, exaltando os riscos que a mesma pode trazer à saúde coletiva. Tais alegações são provenientes do uso distorcido e descontextualizado de informações, além da equivocada argumentação de autonomia privada para a rejeição da interferência do Estado (Gardoni, 2017). Contudo, o quadro não 
configura exatamente uma especificidade da pandemia de COVID-19, mas revela traços de continuidade com eventos passados que trouxeram diversos desafios às políticas públicas de saúde em outras épocas, como foi o caso do que ocorreu na Revolta da Vacina.

\subsection{A vacinação no Brasil e a Revolta de 1904}

Desde o surgimento da primeira vacina, desenvolvida em 1798, pelo médico inglês Edward Jenner, sempre houve objeção à vacinação. Acredita-se que a vacina antivariólica tenha chegado a território nacional pela primeira vez em 1804 . Dom João VI ordenou que a publicação de Edward Jenner sobre a vacina antivariólica fosse traduzida e, em abril de 1811, no Rio de Janeiro, foi formada a Junta da Instituição Vacínica, subordinada, por sua vez, ao Intendente Geral da Polícia. Contudo, a doença também conhecida como "mal das bexigas" retornou de forma marcante e intensa entre os anos de 1834 e 1838. Nesse período, houve a retomada ilegal do tráfico negreiro e inserção furtiva de tais indivíduos na cidade, que permaneciam aninhados em depósitos localizados na região suburbana, em condições desumanas (Chalhoub, 1996).

O primeiro mapa anual de vacinação do Brasil é datado de 1820, no qual consta a imunização de 2688 indivíduos, dos quais 1803 eram escravizados (Chalhoub, 1996). Em contrapartida, no fim de 1830, a vacinação apresentou declínio numérico, de modo que alguns relatórios referenciavam a preocupação da população com horror à vacina, designada de "vacinophobia". Esse temor pode ser explicado pela técnica de imunização utilizada na época, conhecida como vacinação braço a braço. Após 8 dias, os vacinados deveriam retornar ao local, para que o fluido presente nas pústulas fosse extraído e utilizado em outros indivíduos. Porém, muitos sujeitos evitavam a volta por ser um processo incômodo e demorado. Ademais, polêmicas oriundas de Portugal, relacionadas à possibilidade do desenvolvimento de doenças das vacas, assim como a incitação do Clero em afirmar que a vacina era "um presente de Satâ", repercutiram em todo o território brasileiro, afetando diretamente o alastramento da vacinação (Larocca \& Carraro, 2000).

Outro aspecto importante é que havia contradições por parte dos próprios médicos sobre a eficácia da vacina, já que ocorriam casos da doença entre os vacinados. Além disso, o diagnóstico incerto atrelado a outras enfermidades, como a varicela (catapora), dava origem a alegações de que a vacina causava a doença. Para mais, a dificuldade de importação da linfa da Europa e notícias sobre a transmissão de sífilis entre os vacinados braço a braço, estimulavam a inquietação do povo e tornavam o cenário cada vez mais caótico. Por fim, a população confundia a vacinação com a variolização, isto é, a inoculação da varíola benigna, praticada por curandeiros, feiticeiros e homeopatas.

Em 1846, a imunização tornou-se obrigatória em todo o país pelo Decreto Imperial no 464, com o intuito de controlar o número de casos da doença, e na tentativa de difundir a cultura da vacina na população. Já em 1891, o estado de São Paulo tornou a vacina antivariólica compulsória e 10 anos após, o Rio de Janeiro adotou a mesma medida, gerando a revolta do povo, além de abrir debates e polêmicas sobre o respeito às liberdades individuais e as intervenções do Estado a nível de Saúde Pública (Larocca \& Carraro, 2000).

Além do incessante questionamento acerca da forma truculenta de execução do decreto, acreditava-se que os soros e os aplicadores não eram confiáveis, bem como os enfermeiros, fiscais e policiais responsáveis pela campanha de vacinação, pois possuíam instintos brutais e eram dotados de imoralidade (Neves et al., 2020). Vale ressaltar, ainda, que o serviço de vacinação na Corte não conseguiu acompanhar o ritmo de crescimento populacional no Rio de Janeiro, onde em 1880 a população urbana chegou a dobrar numericamente. Tal processo deve-se a alguns fatores, como: o crescimento natural do povo, a abolição da escravidão e a vinda de imigrantes europeus para o Brasil (Neves et al., 2020).

No início do século XX, o intenso aumento demográfico começou a gerar seus primeiros reflexos, desencadeando um conjunto de problemas às famílias mais pobres e vulneráveis. Essas passaram a enfrentar grandes dificuldades relacionadas à falta de moradia, de saneamento básico, de higiene e de abastecimento de água (Neves et al., 2021). Desse modo, o ambiente 
insalubre gerado cooperou para as elevadas taxas de mortalidade das epidemias que assolaram o Brasil. Sendo assim, o combate às epidemias se tornou indispensável para o presidente Rodrigues Alves.

Nessas circunstâncias, o médico sanitarista Oswaldo Cruz e o engenheiro Pereira Passos tornaram-se peças chave em um dos principais objetivos de seu governo: concluir o projeto de urbanização e saneamento do Rio de Janeiro (Gardoni, 2017). Entretanto, a resistência da população carioca ao processo fortaleceu-se ainda mais, mostrando assim a oposição crescente dos cidadãos em relação ao sanitarismo.

Neves destaca dois marcos históricos fundamentais para a oposição ao sanitarismo e para o início dos movimentos antivacina brasileiros: a regulamentação do "código de torturas" e a Revolta da Vacina, ambos ocorridos em 1904 (Neves et al., 2021). No que diz respeito ao primeiro marco, é preciso esclarecer que, com o objetivo de melhorar e defender os interesses da saúde pública, Rodrigues Alves elaborou um regulamento sanitário por meio do Decreto 5.156, de 8 de março de 1904, que ficou popularmente conhecido como "código de torturas", uma vez que seu teor foi considerado arbitrário (Neves et al., 2021).

O Decreto regulamentava de forma detalhada as ações da polícia sanitária nos domicílios e as funções de notificação, isolamento, desinfecções e vigilância médica do serviço de profilaxia geral de moléstias infecciosas, como a febre amarela e a varíola. Tornava possível interditar e desocupar prédios para executar os expurgos, desinfecções e melhoramentos considerados "convenientes", quando fossem registrados casos de contágio, más condições higiênicas ou aglomerações de moradores (artigo 175). Quando os defeitos de higiene fossem considerados "insanáveis", o prédio podia ser demolido (artigo 123). Para eficácia do serviço, o Regulamento mandava que a polícia atendesse a requisições das autoridades sanitárias, sempre que seu auxílio fosse pedido "no interesse da saúde pública" (artigo 303) (Cantisano apud Neves et al., 2021).

Além da resistência do povo atrelada às interferências do Estado na vida privada, os impactos dessas medidas sanitaristas implementadas por Rodrigues Alves e Oswaldo Cruz chocaram-se com a relutância popular, pois "as pessoas desconfiavam das modernas medidas sanitaristas impostas pelo governo Republicano, sentindo medo, subvertendo e fazendo chacota das medidas adotadas pelo sanitarista" (Gardoni, 2017, p. 41). Mesmo assim, o projeto de sanitizar o Rio de Janeiro e erradicar as epidemias se manteve a todo vapor.

Em 9 de novembro de 1904, a regulamentação de aplicação da vacina obrigatória fora publicada. Tal atitude intransigente foi tomada objetivando a contenção dos efeitos aniquiladores da varíola. Todavia, tornou-se o estopim para o evento conhecido como Revolta da Vacina (Neves et al., 2020). Resistindo à imposição da lei, a população tomou as ruas do centro carioca no dia 10 de novembro, em um movimento que perdurou por dias de ações revoltosas (Sevcenko, 2018). Já no dia 14 de novembro de 1904, o jornal Correio da Manhã trazia a respectiva manchete: "Vacinação Obrigatória Reação do Povo", para expor o cenário de miséria e morte em que a população se encontrava. Com a rejeição em massa ao imunizante devido às notícias falsas, a rebelião somente foi controlada quando o governo brasileiro revogou a lei que tornava a vacina obrigatória, expondo de maneira breve os desdobramentos gerados na sociedade pelos movimentos antivacina (Campos, 2017).

\section{2 $O$ retorno dos movimentos antivacina}

Mais recentemente, a divulgação de desinformação e de fake news tem levado ao retorno dos movimentos antivacina, que se alastraram e ganham cada vez mais força, não só no Brasil, mas em todo o mundo. Como resultado, esse processo acaba por diminuir a taxa de vacinação, gerando brecha para a retomada da circulação de doenças infectocontagiosas anteriormente controladas e pondo em risco a saúde coletiva da população. A título de exemplo, na cidade de São Paulo, em 2011, ocorreu um leve surto de sarampo com registro de 27 casos. Nesse sentido, 18 desses evoluíram em ambiente escolar, onde a não vacinação de crianças por decisão familiar abriu uma brecha para a instalação da infecção, além da dispersão do vírus por regiões próximas (Saúde SP, 2011). Ademais, em 2017, o Brasil testemunhou o surto mais intenso de febre amarela desde 
1980. Assim, com receio de que a doença se difundisse ainda mais, o Ministério da Saúde e a Organização Mundial da Saúde (OMS) reforçaram a vacinação em território nacional (Campos, 2017).

Ao longo da história, a ciência vem demonstrando um conjunto de benefícios e avanços que permitem prolongar e assegurar maior qualidade de vida ao ser humano. Nesse âmbito, são exemplos não só o desenvolvimento de vacinas para o combate às moléstias, mas também de fármacos e métodos diagnósticos mais eficazes. Logo, é possível constatar que os avanços científicos e tecnológicos proporcionam o melhoramento das condições de saúde pública, principalmente pelo potencial na prevenção e erradicação de doenças.

No Brasil, a criação do Programa Nacional de Imunizações (PNI) foi um importante marco para a saúde da população. Coordenado pelo Ministério da Saúde, o PNI foi criado em 1973 em conjunto com as secretarias municipais e estaduais de saúde e vem consolidando-se como uma das mais expressivas intervenções em saúde pública do país. O PNI tem mostrado grande eficiência e, por meio do Calendário Nacional de Vacinação (CNV), impacta diretamente no perfil de morbimortalidade da população brasileira, adequando-se às mudanças ocorridas nas esferas política, epidemiológica e social. Parte desse sucesso deve-se ao fato do PNI, como política de Estado, seguir os princípios do Sistema Único de Saúde (SUS), referentes à universalidade e equidade da atenção, assim como ao princípio organizativo de descentralização, com direção única em cada esfera de governo, estabelecidos através da regulamentação do SUS, pela Lei Orgânica da Saúde (Lei nº 8.080), de 1990 (Brasil, 1990).

Dessa forma, o cenário epidemiológico de doenças passíveis de imunoprevenção sofreu grande mudança, firmando o ato vacinal como uma intervenção sanitária imprescindível e trazendo a erradicação da poliomielite, da rubéola, da síndrome da rubéola congênita e do tétano neonatal. Houve também redução radical na ocorrência de outras doenças transmissíveis, como: coqueluche e difteria, responsáveis por dizimar milhares de vidas e deixar sequelas que afetam a qualidade de vida e a saúde do povo brasileiro (Domingues \& Teixeira, 2013). Ademais, o controle de outras infecções em todo o território nacional também se tornou possível graças ao PNI, entre essas a tuberculose, o sarampo, o HPV, o H1N1 e o Haemophilus Influenzae. Há, contudo, algumas doenças que já tinham sido erradicadas antes do PNI, respectivamente, a varíola e a febre amarela urbana. Sendo assim, é notável a importância e grandeza da vacinação para a saúde coletiva no Brasil.

No entanto, mesmo com resultados extremamente significativos em todo o mundo, o dia 26 de fevereiro de 1998 marcou o primórdio de uma incerteza internacional atrelada à vacinação, refletindo-se até os dias atuais. Wakefield e colaboradores (1998) apresentaram uma pesquisa preliminar, publicada pela revista Lancet, relatando 12 crianças com desenvolvimento de comportamentos autistas e inflamação intestinal grave. O estudo dizia que a população amostral apresentava resquícios do vírus do sarampo no organismo. Logo, foi sugerido que a vacina tríplice viral ou MMR (do inglês "measles, mumps and rubella") para o combate do sarampo, caxumba e rubéola predispunha ao quadro de regressão comportamental e ao distúrbio de desenvolvimento generalizado em crianças. Embora o número de indivíduos na amostra fosse pequeníssimo e o desenho do estudo questionável, a especulação sobre as conclusões cresceu e a pesquisa ganhou vasta publicidade. Com isso, as taxas de vacinação MMR tiveram declínio pela preocupação de pais e familiares com o possível "risco de autismo" pós-vacinação (DeStefano \& Chen, 1999).

Subsequentemente, estudos epidemiológicos foram realizados, refutando a relação entre a vacinação MMR e o autismo (Taylor et al., 1999; Dales et al., 2001). Passados alguns anos, houve uma curta retratação associada à interpretação dos resultados, por 10 dos 12 coautores. Segundo esses, não foi definida nenhuma relação de causa/efeito associada à vacina MMR e o autismo, já que os dados eram insuficientes (Murch et al., 2004). Em fevereiro de 2010, a revista retirou o artigo, admitindo claramente que inúmeros elementos do estudo estavam incorretos (Eggertson, 2010). Por fim, os envolvidos no estudo foram considerados culpados por conduta antiética, uma vez que realizaram investigações sem obtenção das autorizações éticas necessárias, e deturpação científica, pela descrição de que a amostragem utilizada na pesquisa foi 
consecutiva, quando na verdade, foi seletiva. Tal retratação foi publicada em nome dos editores da revista, na forma de um pequeno parágrafo anônimo (Anonymous, 2010). Como adendo, foram também culpados por fraude deliberada (Godlee, 2011).

Como consequência, muitas famílias evitaram a vacinação devido à manipulação de resultados, pondo em risco crianças à contração de doenças e suas complicações. No mais, surtos de sarampo ocorridos no Reino Unido em 2008-2009, além das bolsas de sarampo no Canadá e nos Estados Unidos, foram associadas a não vacinação (Eggertson, 2010). Portanto, é possível notar que, o uso errôneo de informações científicas e transmissão dessas à população tende a causar efeitos desastrosos a qualquer momento, em especial quando se diz respeito à imunoprevenção.

\subsection{A pandemia de COVID-19, o movimento antivacinação e as fake news}

Nenhum evento recente gerou tanta desconfiança atrelada à divulgação científica quanto a pandemia de COVID-19. Porém, em tempo recorde as pesquisas resultaram na produção e na distribuição de vacinas, assim como outros insumos necessários ao combate do cenário pandêmico atual. A primeira cooperação global para enfrentar a pandemia teve início em 24 de abril de 2020, com a criação do "The Access to COVID-19 Tools Accelerator", apresentando como objetivo incrementar a produção, desenvolvimento e acesso a possíveis tratamentos, testes e vacinas contra o SARS-CoV-2 (Oms, 2020). A colaboração passou a supervisionar mundialmente todas as pesquisas, a fim de obter uma vacina eficaz.

Desse modo, acompanhados de um setor de desenvolvimento e pesquisa fortificado, países com alto IDH começaram à frente nos processos relacionados à produção, testagem, aplicação de doses, supervisão, divulgação dos resultados referentes à eficácia, e efeitos adversos das vacinas desenvolvidas (Oliveira, 2021). Segundo a Organização Mundial da Saúde, 391.426 estudos sobre o tema já foram realizados mundialmente, enquanto 20 vacinas encontram-se aprovadas ou em avaliação (Who, 2021; Who, 2021). Já no Brasil, 6 dessas estão disponíveis para uso, respectivamente: AstraZeneca, CoronaVac, Covaxin, Janssen, Pfizer e Sputnik V. Entretanto, um pequeno número de indivíduos ainda possui desconfiança acerca das vacinas. Na França, por exemplo, estima-se que $75 \%$ da população sejam a favor da vacinação, mas $16 \%$ não consideram o método seguro (Altay \& Lakhlifi, 2020). Para mais, é importante lembrar que a incredulidade nesse âmbito tende a aumentar e se espalhar com auxílio dos movimentos antivacina.

Assim como ocorreu durante a Revolta da Vacina, atualmente, os grupos contrários à vacinação sustentam o movimento por meio de argumentos de base religiosa, moral e filosófica, apesar de ser possível perceber hoje uma maior adesão de indivíduos de classe social mais alta (Campos, 2017). Vale ressaltar que os movimentos antivacina vêm se fortalecendo pela rápida disseminação de notícias falsas, também conhecidas como fake news, através das mídias sociais. A partir de um ambiente favorável para o surgimento de notícias discordantes, a internet tornou-se um ambiente fértil para campanhas contrárias a vacinação, que, por sua vez, propiciam, na prática, a retomada de doenças controladas ou erradicadas, ou seja, cuja imunoprevenção já se encontra disponível, mas não é aderida por todos (Succi, 2018). No contexto da pandemia de COVID-19, a difusão de informações falsas tem sido considerada uma pandemia paralela, denominada como "infodemia", e que contribui decisivamente para uma maior disseminação do vírus (Patten \& Barberton, 2020).

Nesse contexto, o papel do Estado é coordenar ações que direcionem o comportamento correto dos indivíduos com relação à saúde pública, objetivando a unidade e coesão social e, assim, combatendo a pandemia. Todavia, as fracas e/ou controversas declarações de alguns líderes acabam promovendo desconfiança, incerteza e raiva (The Lancet, 2020). Enquanto inúmeros governos nacionais foram altamente responsivos à ameaça da nova doença, outros desconsideram a pandemia $\mathrm{e}$ resistem em seguir as determinações da Organização Mundial da Saúde (OMS). Dentre os governos relutantes, pode-se citar o do Brasil, no qual o presidente Jair Messias Bolsonaro vem sendo criticado continuamente por especialistas em saúde, e confronta uma forte reação pública (The Lancet, 2020). 
O líder do executivo federal brasileiro considerou e, continua considerando, as ações de restrição de contato interpessoal como algo radical, além de atacar ações decretadas por governadores estaduais (Garrido \& Rodrigues, 2020). Daí deu-se uma eclosão contínua de fake news, especificamente associada à vacinação. Junto com a carência de conhecimento técnico-científico e pensamento crítico, as falácias espalhadas através das redes sociais obscurecem o entendimento da população sobre a relevância da imunização. Contudo, isso ocorre diferentemente do quadro em que se desenhou a Revolta da Vacina, onde havia uma inconsistência científica e as hipóteses acerca da vacinação eram, de fato, controversas até mesmo para os intelectuais. Ao contrário do que se delineou em 1904, atualmente sabe-se que a oposição à vacinação é infundada cientificamente, além do mais, é inegável que o acesso à informação confiável é muito maior.

No tocante à saúde coletiva, a desinformação pode gerar danos irreparáveis, influenciando a visão dos cidadãos em relação à pandemia e, principalmente, indivíduos idosos (Yabrude et al., 2020). Esse ponto é de grande importância, uma vez que esses são mais vulneráveis a notícias falsas, além do que, sujeitos acima de 65 anos de idade são 7 vezes mais propensos a disseminar fake news quando comparados a indivíduos com menos de 29 anos (Yabrude et al., 2020). Entre as hipóteses relacionadas à vulnerabilidade estão: o analfabetismo absoluto e funcional presentes na população idosa, e o posicionamento político-comportamental (Guess et al., 2019). Além disso, o provável uso de bots (robôs) associado à automatização do compartilhamento massivo de informações, através do uso de contas de usuários(as), potencializa a velocidade, a eficácia e o alcance de conteúdos falsos e/ou distorcidos, propiciando dessa maneira o aumento do número de fake news (Bucci, 2018; Teixeira \& Couto Junior, 2020). Consequentemente, a forma como o cidadão enxerga a realidade, atrelada às notícias falsas sobre a pandemia de COVID-19, e principalmente acerca da vacinação, é influenciada pelas interações ocorridas no cotidiano.

Um estudo realizado pela Fundação Oswaldo Cruz, em conjunto com 25 mil profissionais da saúde em todo o Brasil, demonstrou que $90 \%$ desses veem a difusão de fake news como percalço no combate à doença e à pandemia (Leonel, 2021). Assim, a necessidade de ações governamentais para incentivar a proteção através das vacinas é primordial, de modo que a confiança pública deve ser conquistada por meio de intervenções transparentes embasadas em evidências científicas e comunicação firmada em fatos (Leward \& Lo, 2020). Sobre esse aspecto, chama atenção o fato de que antes da população carioca tomar as ruas do Rio de Janeiro na ação revoltosa de 1904, algumas colunas e manchetes do periódico Correio da Manhã passaram a promover uma campanha contra a obrigatoriedade da vacinação. Nesse ínterim, constantemente via-se a vacina sendo retratada como propagadora de moléstias, destruidora da saúde e causadora de morte (Correio da Manhã, 1904). Dessa forma, o passado demonstra que as consequências de reiteradas declarações contra a vacinação, pautadas em argumentos falaciosos, além de prejudicar o controle de epidemias e desinformar a população, também são capazes de instaurar o caos e promover a desobediência civil.

Infelizmente, no Brasil percebe-se um regresso do obscurantismo que, associado a políticas do atual governo, dificulta o combate pandêmico. A interrupção da divulgação do número de mortes por COVID-19, por exemplo, destaca o bloqueio das informações à população, arrevesando a difusão das notícias e o conhecimento dos indivíduos acerca da realidade atual. Nesse âmbito, a imposição de obstáculos à compra e distribuição de imunizantes é um fator de grande limitação, conforme vem sendo apurado por meio de uma Comissão Parlamentar de Inquérito (CPI) (Bbc Brasil, 2021). Para além disso, as declarações do Presidente da República pondo à prova a segurança e eficácia das vacinas tiveram grande repercussão, principalmente nas classes mais baixas, levando a um efeito cascata e potencializando as incertezas, desconfianças e, até mesmo, a negação do imunizante pelo povo. Dentre essas, podem ser destacadas as falas de que "o povo brasileiro não será cobaia de ninguém" e que após a vacinação, os imunizados poderiam "virar jacaré" (IstoÉ, 2020; Monari et al., 2021), tal qual aconteceu no século XIX, quando chegou-se a disseminar a ideia de que os vacinados contra a varíola se transformariam em vacas ou bois (Neves et al., 2021). 
Apesar dos desafios impostos às políticas públicas pelas informações falsas, muitas repercutidas por parte do governo brasileiro, a maior parte da população crê na vacinação contra esta nova doença emergente. É o que demonstra um estudo publicado na revista Nature, segundo o qual, dos 19 países mais afetados pela COVID-19, o Brasil foi o segundo colocado no ranking de aceitação das vacinas pela população, respectivamente $85,3 \%$; enquanto o primeiro colocado foi a China, com 88,6\% (Lazarus et al., 2021). Em busca de atender a essa demanda, o Ministério da Saúde elaborou o plano de vacinação contra a COVID-19 (Ministério da Saúde, 2020), embora a velocidade da vacinação pudesse ser maior, uma vez que já foram noticiadas muitas falhas no que se refere à gestão governamental. É o caso da demora na contratação de empresas produtoras de agulhas e seringas; acontecimentos diplomáticos envolvendo países produtores de vacinas e outros insumos; inépcia nos acordos com laboratórios responsáveis pela fabricação de imunizantes para abastecimento do SUS; além do atraso na distribuição dos lotes de vacinas; impactando assim a disponibilidade do imunizante em todo o território nacional (Castro, 2021).

De toda forma, a política de imunização contra o coronavírus depende de esforços no combate contra as fake news, com expansão de informações confiáveis e campanhas de conscientização sobre a temática (Leward \& Lo, 2020; Szwarcwald, 2020). Além disso, outras formas de estímulo à vacinação podem ser colocadas em prática, como a instituição de penalidades aos que resistem à adesão da imunoprevenção obrigatória. A rígida exigência do passaporte de vacina para a realização de viagens, assim como para a entrada e permanência em certos ambientes também podem ser de grande auxílio à imunização. Logo, o processo de locomoção de tais indivíduos seria estritamente reduzido, não deixando outra escolha senão o aceite da vacina.

\section{Considerações Finais}

A partir da análise histórica, evidencia-se que o movimento contra a vacinação está presente no Brasil há mais de um século, fato que pode ser comprovado pelos episódios em torno da Revolta da Vacina. Apesar do indelével papel da imunização na prevenção ao contágio de doenças transmissíveis, certos grupos insistem em se posicionar contra a vacinação, confrontando a ciência com argumentos falaciosos de todo tipo, de modo que os impactos causados pelos antivacinas podem ser observados nos novos surtos de doenças que já se mostravam controladas, como o sarampo.

No Brasil, principalmente a partir da década de 1970, o Programa Nacional de Imunizações estabelece importante marco para a saúde da população, especialmente quando um grande número de pessoas ainda vive em condições de carência associadas ao saneamento básico, acesso a diagnóstico e tratamento muito restrito. No entanto, tal fato parece irrelevante para aqueles que, tal como se deu no passado, alegam a autonomia privada como justificativa da oposição ao processo de vacinação. Recentemente, a pandemia de COVID-19 fez com que esses discursos ressurgissem através do uso das mídias sociais como ferramenta para a disseminação de notícias falsas. No âmbito brasileiro, muitas dessas notícias foram originadas no governo federal, obscurecendo o entendimento dos cidadãos e gerando confusão entre as informações verdadeiras e falsas, em especial quando se diz respeito à imunoprevenção. Desse modo, estimula-se ainda mais os movimentos antivacinas, levando à desconfiança e queda da taxa de vacinação não só associada ao cenário atual, mas também a outras doenças previamente controladas.

Tal qual ocorreu durante a Revolta da Vacina, o movimento antivacina, além de se alicerçar em bases religiosas, morais e jurídicas, continua associando a vacinação às doenças e a perda de saúde. Logo, é possível notar que o contexto em que se deu à revolta e o contexto da pandemia do COVID-19 possuem semelhanças sutis e também excessivas, como é o caso da colocação de que a vacina transforma os imunizados em animais. Contudo, mesmo que ainda exista carência de conhecimento técnico-científico por parte de algumas pessoas que refutam a vacinação, sabe-se que a oposição à vacina é infundada cientificamente, além do mais, é inegável que o acesso à informação confiável é muito mais amplo e democrático 
quando comparado ao ano de 1904. Dessa forma, é possível observar como a deturpação de informações que, no passado, chegou a levar a população carioca a se levantar contra as medidas sanitárias em um episódio marcante de desobediência civil, hoje afeta diretamente a imunização da população e coloca a saúde coletiva em risco.

Portanto, foi percebida uma linha de continuidade entre os discursos do passado e do presente que se opõem às medidas restritivas e à vacinação, tanto sob a alegação de defesa da liberdade individual, quanto pela invocação de ideias obscurantistas. Conclui-se que a recusa arbitrária ao cumprimento de políticas públicas sanitárias configura exercício abusivo do direito de liberdade individual, por expor a sociedade a riscos graves e evitáveis.

\section{Referências}

Anonymous. (2010). Retraction-Ileal-lymphoid-nodular hyperplasia, nonspecific colitis, and pervasive developmental disorder in children. Lancet, 375(9713), 445. Doi: 10.1016/S0140-6736(10)60175-4.

Altay, S., \& Lakhlifi, C. (2020). Are science festivals a good place to discuss heated topics? Journal of Science Communication, 19(1), A07. https://jcom.sissa.it/archive/19/01/JCOM_1901_2020_A07.

Bbc Brasil. (2021). CPI da covid: quem é quem no escândalo Covaxin. https://www.bbc.com/portuguese/brasil-57647163.

Brasil. (1990). Lei n o 8080, de 19 de setembro de 1990. Dispõe sobre as condições para a promoção, proteção e recuperação da saúde, a organização e o funcionamento dos serviços correspondentes e de outras providências. Diário Oficial da União. http://conselho.saude.gov.br/legislacao/lei8080_190990.htm\#: :text=LEI\%20N\%C2\%BA\%208.080\%2C\%20DE\%2019\%20DE\%20SETEMBRO\%20DE,Nac ional $\% 20$ decreta $\% 20 \mathrm{e} \% 20 \mathrm{eu} \% 20$ sanciono $\% 20 \mathrm{a} \% 20$ seguinte $\% 201 \mathrm{ei} \% 3 \mathrm{~A}$.

Bucci, E. (2018). Pós-política e corrosão da verdade. São Paulo. Revista USP, 116, 19-30. Doi: https://doi.org/10.11606/issn.2316-9036.v0i116p19-30.

Campos, A. L. (2017). Uma análise bioética das decisões judiciais brasileiras sobre os eventos adversos pós-vacinação. Tese (Doutorado em Bioética)— Universidade de Brasília. Brasília. https://repositorio.unb.br/handle/10482/23893.

Cantisano, P. J. (2015). Lares, tribunais e ruas: a inviolabilidade de domicílio e a Revolta da Vacina. Revista Direito e Práxis, 6(11), 294-325. Doi: $10.12957 /$ dep.2015.16529.

Castro, R. (2021). Vacinas contra a Covid-19: o fim da pandemia? Revista de Saúde Coletiva, 31(1), e310100. Doi: http://dx.doi.org/10.1590/S010373312021310100 .

Chalhoub, S. (1996). Cidade febril. São Paulo: Companhia das Letras. http://repositoriolabim.cchla.ufrn.br/bitstream/ 123456789/733/1/CORTI\%C3\%87OS.\%20Cidade\%20febril\%20corti\%C3\%A7os\%20e\%20epidemias\%20na\%20corte\%20imperial.\%20CHALHOUB\%2C\% 20Sidney.\%201996.pdf.

Correio Da Manhã. (1904). Acervo Digital Biblioteca. http://hemerotecadigital.bn.br/acervo-digital/correio-manha/089842.

Dales, L., Hammer, S. J., \& Smith, N. J. (2001). Time trends in autism and in MMR immunization coverage in California. JAMA, $285(9)$, 1183-1185. https://jamanetwork.com/journals/jama/articlepdf/193604/JBR00284.pdf.

DeStefano, F., \& Chen, R. T. (1999). Negative association between MMR and autism. Lancet, 353(9169), 1987-1988. Doi: 10.1016/S0140-6736(99)00160-9.

Domingues, C. M. A. S., \& Teixeira, A. M. S. (2013). Coberturas vacinais e doenças imunopreveníveis no Brasil no período 1982-2012: avanços e desafios do Programa Nacional de Imunizações. Epidemiol Serv Saúde, 22(1), 9-27. Doi: 10.5123/S1679-49742013000100002.

Eggertson, L. (2010). Lancet retracts 12-year-old article linking autism to MMR vaccines. CMAJ, 182(4), E199-200. Doi: 10.1503/cmaj.109-3179.

Gardoni, R. K. (2017). Vacinação obrigatória: liberdade individual e utilidade pública na modernização do direito brasileiro na república velha. Trabalho de Conclusão de Curso. Universidade Federal do Paraná. Curitiba. https://acervodigital.ufpr.br/bitstream/handle/1884/58176/RENNAN\% 20KLINGELFUS\%20GARDONI.pdf?sequence=1\&isAllowed=y AN KLINGELFUS GARDONI.pdf.

Garrido, R. G., \& Rodrigues, R. C. (2020). Restrição de contato social e saúde mental na pandemia: possíveis impactos das condicionantes sociais. $J$ Health Biol Sci, 8(1), 1-9. Doi: 10.12662/2317-3325jhbs.v8i1.3325.p1-9.2020.

Guess, A., Nagler, J., \& Tucker J. (2019). Less than you think: pevalence and predictors of fake news dissemination on Facebook. Sci Adv, 5(1), eaau4586. Doi: https://doi.org/10.1126/sciadv.aau4586.

Godlee, F. (2011). The fraud behind the MMR scare. BMJ, 342, d22. Doi: 10.1136/bmj.d22. 
IstoÉ. (2020). Bolsonaro sobre vacina da Pfizer: 'Se você virar um jacaré, é problema seu'. https://istoe.com.br/bolsonaro-sobre-vacina-de-pfizer-se-vocevirar-um-jacare-e-problema-de-voce.

Jesus, C. S. de., Rezende, M. F. M., Dias, A. K., Couto, G. B. F. do., Markus, G. W. S., \& Pereira, R. A. (2021). O papel da Agência Nacional de Vigilância Sanitária na regulação de vacinas. Research, Society and Development, 10(6), e35010614512. https://doi.org/10.33448/rsd-v10i6.14512.

Larocca, L. M., \& Carraro, T. E. (2000). O mundo das vacinas - caminhos (des)conhecidos. Cogitare Enferm, 5(2), 43-50. Doi: http://dx.doi.org/10.5380/ce.v5i2.44884.

Lazarus, J. V., Ratzan, S. C., Palayew, A., Gostin, L. O., Larson, H. J., Rabin, K., Kimball, S., \& El-Mohandes, A. (2021). A global survey of potential acceptance of a COVID-19 vaccin. Nat Med, 27(2), 225-228. Doi: 10.1038/s41591-020-1124-9.

Leonel, F. (2021). Pesquisa analisa o impacto da pandemia entre profissionais de saúde. [Internet]. Rio de Janeiro: Portal FIOCRUZ. https://portal.fiocruz.br/noticia/pesquisa-analisa-o-impacto-da-pandemia-entre-profissionais-de-saude.

Leward, J. A., \& Lo, N. C. Scientific and ethical basis for social-distancing interventions against COVID-19. Lancet Infect Dis, 20(6), 631-633. Doi: $10.1016 / \mathrm{S} 1473-3099(20) 30190-0$

Ministério da Saúde. Secretaria de Vigilância em Saúde. Departamento de Imunização e Doenças Transmissíveis. Coordenação-Geral do Programa Nacional de Imunizações. (2020). PLANO NACIONAL DE OPERACIONALIZAÇÃO DA VACINAÇÃO CONTRA A COVID-19. https://www.gov.br/saude/ptbr/media/pdf/2020/dezembro/16/plano_vacinacao_versao_eletronica.pdf ano_vacinacao_versao_eletronica.pdf.

Monari, A. C. P., Araújo , K. M. de, Souza, M. R. de, \& Sacramento, I. (2021). Disputas narrativas e legitimação: análise dos argumentos de Bolsonaro sobre vacinação contra Covid-19 no Twitter. Liinc Em Revista, 17(1), e5707. Doi: https://doi.org/10.18617/liinc.v17i1.5707.

Murch, S. H., Anthony, A., Casson, D. H., Malik, M., Berelowitz, M., Dhillon, A. P., et al. (2004). Retraction of an interpretation. Lancet, 363(9411), 750. Doi: 10.1016/S0140-6736(04)15715-2.

Neves, R. A., Gomes, D.M., Fernandes, C. M. F. L., Cicilio, T. S.(2020). O discurso de resistência na Revolta da Vacina e os direitos civis. In: ROSA, A. et. al (Org). Direitos Humanos e Fundamentais. Rio de Janeiro: Pembroke Collins. https://www.academia.edu/44826784/Livro_Direitos_Humanos_e_Fundamentais_Vol_2p.

Neves, R. A., Gomes, D. M., Soares, Y. E., Oliveira, C. P. R. (2021). De volta para o passado: o movimento antivacina e as políticas públicas de imunização no Brasil. In ASENSI, Felipe et. al (Org). Políticas públicas e saúde. Rio de Janeiro: Pembroke Collins. https://www.academia.edu/45000860/A_pandemia_de_COVID_19_e_a_flexibiliza\%C3\%A7\%C3\%A3o_da_Lei_de_Responsabilidade_Fiscal.

Oliveira, F. (2021). 14 vacinas aprovadas para Covid-19 e dezenas em teste em todo o mundo; conheça. https://www.cnnbrasil.com.br/saude/2021/04/24/saibaquais-sao-as-vacinas-contrao-novo-coronavirus-em-uso-e-em-estudo-no-mundo.

Organização Mundial Da Saúde - OMS. (2020). Concepção da OMS sobre acesso justo e distribuição equitativa de produtos de saúde contra a COVID-19. https://www.who.int/docs/default-source/coronaviruse/202485-covid19-e-2-pt.pdf.

Patten, S., \& Barberton, Z. (2020). Infodemic: Coronavirus and the fake news pandemic. Nature. [internet] 2020 [acesso em 2021 set 13]. Doi: $10.1038 / \mathrm{d} 41586-020-01409-2$.

Pereira, A. S., et al. (2018). Metodologia da pesquisa científica. UFSM. https://repositorio.ufsm.br/bitstream/handle/1/15824/Lic_Computacao_Metodologiapesquisa-Cientifica.pdf?sequence=10_Metodologia-pesquisa-Cientifica.pdf.

Saúde SP. (2011). Coordenadoria de Controle de Doenças. Governo do Estado de São Saulo. [Online].; 2011. [cited 2021 09 07]. http://www.saude.sp.gov.br/coordenadoria-de-controle-dedoencas/aconteceu/alerta-sarampo.

Sevcenko, N. (2018) A Revolta da Vacina: mentes insanas em corpos rebeldes. UNESP. São Paulo.

Succi, R. C. M. (2018). Vacinal Refuse - what we need to know. J. Pediatr. (Rio J.), 94(6), 574-581. Doi: https://doi.org/10.1016/j.jped.2018.01.008.

Szwarcwald, C. L., et al. (2021). ConVid - Pesquisa de Comportamentos pela internet durante a pandemia de COVID-19 no Brasil: Concepção e metodologia de aplicação. Cadernos de Saúde Pública, 37(3), e00268320. Doi: 10.1590/0102-311X00268320.

Taylor, B., Miller, E., Farrington, C. P., Petropoulos, M. C., Favot-Mayaud, I., Li, J., et al. (1999). Autism and measles, mumps, and rubella vaccine: No epidemiologic evidence for a causal association. Lancet, 353(9169), 2026-2029. Doi: 10.1016/S0140-6736(99)01239-8.

Teixeira, M. M., \& Couto Junior, D. R. (2020). Deu ruim na hashtag! bots e pandemia de fake news em tempos de covid-19: o caso \#fechadocombolso(1)naro. revista Interinstitucional Artes de Educar, 6(2), 328-347. Doi: 10.12957/riae.2020.51544.

The Lancet. (2020). COVID-19: learning from experience. Lancet, 395(10229), 1011. Doi: 10.1016/S0140-6736(20)30686-3.

Wakefield, A. J., Murch, S. H., Anthony, A., Linnell, J., Casson, D. M., Malik, M., et al. (1998). Ileal-lymphoid-nodular hyperplasia, non-specific colitis, and pervasive developmental disorder in children. Lancet, 351(9103), 637-641. Doi: 10.1016/s0140-6736(97)11096-0.

Who. (2021). https://search.bvsalud.org/global-literature-on-novel-coronavirus-2019-ncov/?u_filter\%5B \%5D=fulltext\&u_filter\% 5B\%5D=db\&u_filter\% 5B\% $5 \mathrm{D}=$ mj_cluster\&u_filter $\% 5 \mathrm{~B} \% 5 \mathrm{D}=$ type_of_study\&u_filter $\% 5 \mathrm{~B} \% 5 \mathrm{D}=$ clinical_aspect\&u_filter $\% 5 \mathrm{~B} \% 5 \mathrm{D}=1 \mathrm{a} \& \mathrm{u} \_$filter $\% 5 \mathrm{~B} \% 5 \mathrm{D}=\mathrm{year}$ _cluster\&u_filter $\% 5 \mathrm{~B} \% 5$ $\mathrm{D}=$ type\&u_filter\%5B\%5D=ta_cluster\&fb=db\%3A100\&where=\&range_year_start=\&range_year_end=\&range_year_start=\&range_year_end=\#db $>$. Acesso em 6 nov 2021. 
Research, Society and Development, v. 10, n. 14, e487101422476, 2021

(CC BY 4.0) | ISSN 2525-3409 | DOI: http://dx.doi.org/10.33448/rsd-v10i14.22476

Who. (2021). Status of COVID-19 Vaccines within WHO EUL/PQ evaluation process. https://extranet.who.int/pqweb/sites/default/files/ documents/ Status_COVID_VAX_19August2021.pdf>.

Yabrude, A. T. Z., Souza, A. C. M., Campos, C. W., Tiboni, L. B. M. (2020). Desafios das Fake News com idosos durante infodemia sobre Covid-19: experiência de estudantes de medicina. Revista Brasileira de Educação Médica, 44(1) e0140. Doi: https://doi.org/10.1590/1981-5271v44.supl.1-20200381. 